\title{
$2^{2^{\omega}}$ NONISOMORPHIC SHORT ORDERED COMMUTATIVE DOMAINS WHOSE QUOTIENT FIELDS ARE LONG
}

\author{
KRZYSZTOF CIESIELSKI
}

(Communicated by Andreas R. Blass)

\begin{abstract}
A linearly ordered set is short if it does not contain any monotonic sequence of length $\omega_{1}$, and it is long if it contains a monotonic sequence of length $\alpha$ for every ordinal $\alpha<\left(2^{\omega}\right)^{+}$. We prove that there exists a family $\mathbf{F}$ of power $2^{2^{\omega}}$ of long ordered fields of size $2^{\omega}$ that are pairwise nonisomorphic (as fields) and such that every field $F \in \mathrm{F}$ has $2^{2^{\omega}}$ nonisomorphic short subdomains whose field of quotients is $F$. The generalization of this result for higher cardinals is also discussed. This generalizes the author's result of [Ci].
\end{abstract}

\section{Definitions AND NOTATION}

Our set theoretic and algebraic notation is standard and follows [Je] and [La] respectively.

Ordinals are identified with the sets of their predecessors and cardinals with initial ordinals. For an ordinal $\alpha$ we denote by $\omega_{\alpha}$ the $\alpha$ th infinite cardinal, and by $\alpha+1$ the ordinal successor of $\alpha$. For a cardinal $\kappa$ we denote by $2^{\kappa}$ the cardinality of the power set $\mathbf{P}(\kappa)$ and by $\kappa^{+}$the cardinal successor of $\kappa$. Symbols $\mathbf{N}, \mathbf{Q}$, and $\mathbf{R}$ stand for the sets of natural, rational, and real numbers, respectively. For an ordered semigroup $G=\langle G,+, 0\rangle$ we define $G^{+}=\{g \in G: g \geq 0\}$. For a commutative domain $D$ we also use the following notation connected with the existence of radicals

$$
\operatorname{rad}(D)=\left\{d \in D:(\forall n \in \mathbf{N})(\exists y \in D)\left(y^{n}=d\right)\right\} .
$$

Let $X$ and $Y_{x}$ be linearly ordered sets such that $\mathbf{N} \subset Y_{x}$ for all $x \in X$. We define the support of $f \in \prod_{x \in X} Y_{x}$ by $\operatorname{supp}(f)=\{x \in X: f(x) \neq 0\}$ and the direct sum of the family $\left\{Y_{x}\right\}_{x \in X}$ by $S\left(X,\left\{Y_{x}\right\}_{x \in X}\right)=\left\{f \in \prod_{x \in X} Y_{x}: \operatorname{supp}(f)\right.$ is finite . (It is usually denoted as $\sum_{x \in X} Y_{x}$, but in this paper such a notation would lead to very complicated formulas.) In the case when $Y_{x}=Y$ for all $x \in X$ we define $S(X, Y)=S\left(X,\left\{Y_{x}\right\}_{x \in X}\right)$.

Received by the editors April 12, 1989 and, in revised form, December 3, 1990.

1980 Mathematics Subject Classification (1985 Revision). Primary 03E05; Secondary 06F25, $12 \mathrm{~J} 15$.

Key words and phrases. Nonisomorphic, ordered commutative domains, quotient fields. 
A zero element of $S\left(X,\left\{Y_{x}\right\}_{x \in X}\right)$ is defined as the unique element $0 \in$ $S\left(X,\left\{Y_{x}\right\}_{x \in X}\right)$ such that $\operatorname{supp}(0)=\varnothing$. For $x \in X$ we put $e_{x} \in S\left(X,\left\{Y_{x}\right\}_{x \in X}\right)$ such that $\operatorname{supp}\left(e_{x}\right)=\{x\}$ and $e_{x}(x)=1$. Moreover, if $0 \in X$ then every element $y \in Y_{0}, y \neq 0$, is identified with $y=f_{y} \in S\left(X,\left\{Y_{x}\right\}_{x \in X}\right)$, where $\operatorname{supp}\left(f_{y}\right)=\{0\}$ and $f_{y}(0)=y$. In particular, $1=f_{1}=e_{0}$.

The family $S\left(X,\left\{Y_{x}\right\}_{x \in X}\right)$ is ordered antilexicographicaly, i.e., by the formula

$$
f<g \text { if and only if } f(m)<g(m) \text {, where } m=\max \{x: f(x) \neq g(x)\} .
$$

\section{Main Lemmas}

In what follows we use the following easily verified well-known facts. (See e.g. [Ci, Lemma 1].)

Proposition 1.1. Let $S=S\left(X,\left\{Y_{x}\right\}_{x \in X}\right)$, where $X$ and $Y_{x} \supset \mathbf{N}$, for $x \in X$, are linearly ordered sets. Then

(1) the linearly ordered set $\left\{e_{x} \in S: x \in X\right\}$ is order isomorphic with $X$;

(2) if $\left\langle Y_{x},+\right\rangle$ are ordered commutative semigroups for $x \in X$ then so is $S$, where $(f+g)(x)=f(x)+g(x)$ for all $f, g \in S$ and $x \in X$;

(3) if $\left\langle Y_{x},+\right\rangle$ are ordered commutative groups for $x \in X$ then so is $S$; moreover, if all $Y_{x}$ 's are divisible then so is $S$;

(4) if $\langle X,+\rangle$ is an ordered commutative semigroup, $\langle Y,+, \cdot\rangle$ is an ordered commutative domain, and $\left\langle Y_{x},+, \cdot\right\rangle=\langle Y,+, \cdot\rangle$ for every $x \in X$, then $S$ is an ordered commutative domain (sometimes denoted by $X(Y)$ ), where the product between $p, q \in S$ is defined by usual convolution of polynomial multiplication:

$$
(p \cdot q)(x)=\sum\left\{p\left(x_{1}\right) \cdot q\left(x_{2}\right): x_{1}, x_{2} \in X \text { and } x_{1}+x_{2}=x\right\} .
$$

We also use the following fact.

Proposition 1.2. Let $D$ be an ordered commutative domain such that $D$ contains an increasing sequence of length $2^{\omega}+1$. Then, every nontrivial interval of $D$ is long.

Proof. It is easy to prove by transfinite induction on $\alpha<\left(2^{\omega}\right)^{+}$that for every $a, b \in D, a<b$, there is an increasing sequence of type $\alpha$ in the interval $[a, b]$. This implies Proposition 1.2.

Let us also quote the following proposition, which is needed later. An easy proof can be found in [Ci, Lemma 5] or [Ha, p. 6].

Proposition 1.3. If $X$ and $Y_{x}$ are short linearly ordered sets for all $x \in X$ then so is the direct sum $S\left(X\left\{Y_{x}\right\}_{x \in X}\right)$.

Now, let $X$ be a linearly ordered set, let $K$ be a field and define an ordered domain $D$ by $D=S(S(X, \mathbf{N}), K)$. If $\mathbf{Y}=\left\{Y_{x}: x \in X\right\}$ is a set of different variables and

$$
D^{\prime}=K[\mathbf{Y}]=\bigcup\left\{K\left[Y_{x_{1}}, Y_{x_{2}}, \ldots, Y_{x_{n}}\right]: n \in \mathbf{N} \text { and } x_{1}, x_{2}, \ldots, x_{n} \in X\right\} \text {, }
$$


then $D$ and $D^{\prime}$ are isomorphic (as rings). More precisely, an isomorphism $j: D \rightarrow D^{\prime}$ is defined by

$$
\begin{array}{r}
j(f)=\sum\left\{f(g) \cdot\left(Y_{x_{1}}\right)^{g\left(x_{1}\right)} \cdots \cdots\left(Y_{x_{n}}\right)^{g\left(x_{n}\right)}: g \in \operatorname{supp}(f)\right. \\
\text { and } \left.\operatorname{supp}(g)=\left\{x_{1}, x_{2}, \ldots, x_{n}\right\}\right\} .
\end{array}
$$

From the above isomorphism and the theorem that $K\left[X_{1}, \ldots, X_{n}\right]$ is a unique factorization domain (see e.g. [La]) we can easily conclude

Proposition 1.4. Let $D=S(S(X, \mathbf{N}), K)$, where $X$ is a linearly ordered set and $K$ is a field. Then

(1) $D$ is a unique factorization domain;

(2) an element $x \in D$ is invertible in $D$ (i.e., $x^{-1}$ exists in $D$ ) if and only if $x \in K \backslash\{0\}$.

We also need the following lemma.

Lemma 1.5. Let $X$ be a linearly ordered set, $K$ be a field, and let $D=$ $S(S(X, \mathbf{N}), K)$. If $L$ is the quotient field of $D$ then $\operatorname{rad}(L)=\operatorname{rad}(K)$.

In particular, if $K$ is real closed then $\operatorname{rad}(L)=K^{+}$.

Proof. Inclusion " $\supset$ " is obvious.

To prove the converse inclusion let $x \in \operatorname{rad}(L)$ and let us assume $x \neq 0$.

By Proposition 1.4(1), every element of $L \backslash\{0\}$ can be represented as $c p_{1} p_{2} \cdots p_{k} / q_{1} q_{2} \cdots q_{m}$, where $c$ is invertible in $D, p_{1}, p_{2}, \ldots, p_{k}, q_{1}$, $q_{2}, \ldots, q_{m}$ are irreducible elements of $D$, and the number $k+m$ from the above representation is the smallest possible; i.e., the elements $p_{i}$ and $q_{j}$ are not associated for every $i$ and $j$. (Recall that $p, q \in D$ are associated if there exists an invertible element $d$ in $D$ such that $d \cdot p=q$.)

Let $x=c p_{1} p_{2} \cdots p_{k} / q_{1} q_{2} \cdots q_{m}$ be a representation of $x$ as above. Choose arbitrary $n>k+m$ and let $y \in L$ be such that $y^{n}=x$. Moreover, also let $y=d a_{1} a_{2} \cdots a_{s} / b_{1} b_{2} \cdots b_{t}$ be an irreducible representation as above. Hence, $x=y^{n}=d^{n} v_{1} v_{2} \cdots v_{n s} / w_{1} w_{2} \cdots w_{n t}$, where the elements $v_{i}$ and $w_{j}$ are not associated for every $i$ and $j$. So, $k+m=n(s+t)$, which implies that $k=m=s=t=0$.

In other words we proved that for an arbitrary $n>0$ there exists an invertible element $d$ in $D$ such that $x=d^{n}$. But by Proposition 1.4(2), $d \in K$. Hence $x \in \operatorname{rad}(K)$.

\section{CONSTRUCTION OF THE FAMILY OF FIELDS}

Let $\mathbf{B}$ be a transcendence base of $\mathbf{R}$ over $\mathbf{Q}$ and let $\left\{B_{\zeta}: \zeta<2^{2^{\omega}}\right\}$ be an enumeration without repetition of all subsets of $\mathbf{B}$ of cardinality $2^{\omega}$. Moreover, let $\mathbf{Q}\left(B_{\zeta}\right)$ stand for an algebraic closure of $\mathbf{Q} \cup B_{\zeta}$ in $\mathbf{R}$. For $\zeta<2^{2^{\omega}}$ let $E_{\zeta}=$ $S\left(S\left(2^{\omega}+1, \mathbf{N}\right), Q\left(B_{\zeta}\right)\right)$, an ordered commutative domain. Define $K_{\zeta}$ as the quotient field of $E_{\zeta}$, and let $F_{\zeta}$ be the quotient field of $D_{\zeta}=S\left(S(\mathbf{R}, \mathbf{N}), K_{\zeta}\right)$.

We define $\mathbf{F}=\left\{F_{\zeta}: \zeta<2^{2^{\omega}}\right\}$. 
Proposition 2.1. The field $F_{\zeta}$ is long for every $\zeta<2^{2^{\omega}}$.

Proof. Let us fix $\zeta<2^{2^{2}}$. Applying Proposition 1.1(1) twice for pair $2^{\omega}+1$ and $S\left(2^{\omega}+1, \mathbf{N}\right)$ and for the pair $S\left(2^{\omega}+1, \mathbf{N}\right)$ and $E_{\zeta}$, we conclude that $E_{\zeta}$ has an increasing subsequence of length $2^{\omega}+1$. So by Proposition 1.2, $E_{\zeta}$ is long. But $E_{\zeta} \subset K_{\zeta} \subset F_{\zeta}$. Thus, every $F_{\zeta}$ is long.

Proposition 2.2. The fields $F_{\zeta}$ and $F_{\xi}$ are nonisomorphic for every $\zeta<\xi<2^{2^{\omega}}$. Proof. Let us assume by way of contradiction that there exists a field isomorphism $j: F_{\zeta} \rightarrow F_{\xi}$ for some $\zeta<\xi<2^{2^{\omega}}$. Thus by Lemma 1.5,

$$
\begin{aligned}
j\left(\mathbf{Q}\left(B_{\zeta}\right)^{+}\right)=j\left(\operatorname{rad}\left(\mathbf{Q}\left(B_{\zeta}\right)\right)\right) & =j\left(\operatorname{rad}\left(K_{\zeta}\right)\right)=j\left(\operatorname{rad}\left(F_{\zeta}\right)\right)=\operatorname{rad}\left(j\left(F_{\zeta}\right)\right) \\
& =\operatorname{rad}\left(F_{\xi}\right)=\operatorname{rad}\left(K_{\xi}\right)=\operatorname{rad}\left(\mathbf{Q}\left(B_{\xi}\right)\right)=\mathbf{Q}\left(B_{\xi}\right)^{+} .
\end{aligned}
$$

Hence $j\left(\mathbf{Q}\left(B_{\zeta}\right)\right)=\mathbf{Q}\left(B_{\xi}\right)$, which is a contradiction since $\mathbf{Q}\left(B_{\zeta}\right)$ and $\mathbf{Q}\left(B_{\xi}\right)$ are different real closed subfields of $\mathbf{R}$ and hence are not isomorphic. Thus $F_{\zeta}$ and $F_{\xi}$ are nonisomorphic.

Let us also notice that in the Proposition 2.2 we can talk about fields isomorphisms rather that about ordered fields isomorphisms, since all fields $\mathbf{Q}\left(B_{\zeta}\right)$ are real closed and therefore, their ordered structure can be recovered from their algebraic structure.

\section{CONSTRUCTION OF THE FAMILY OF SHORT COMMUTATIVE DOMAINS}

Let us fix $\zeta<2^{2^{\omega}}$. We construct $2^{2^{\omega}}$ nonisomorphic short subdomains $\left\{P_{\eta}: \eta<2^{2^{\omega}}\right\}$ of $F_{\zeta}$ such that $F_{\zeta}$ is a quotient field of each $P_{\eta}$.

Let $\left\{C_{\eta}: \eta<2^{2^{\omega}}\right\}$ be an enumeration, without repetition, of all subsets of $B_{\zeta}$, and let $i$ be a bijection between $\mathbf{R}$ and $E_{\zeta}$. Moreover, for $\eta<2^{2^{\omega}}$ and a finite set $A \subset \mathbf{R}$ let $E_{\eta, A}$ be a subdomain of $E_{\zeta}$ generated by $\mathbf{Q}\left(C_{\eta}\right) \subset E_{\zeta}$ and the set $\{i(r): r \in A\} \subset E_{\zeta}$. In particular, $E_{\eta, \varnothing}=\mathbf{Q}\left(C_{\eta}\right)$. Let $G=S(\mathbf{R}, \mathbf{N})$, and let us define

$$
P_{\eta}=S\left(G,\left\{E_{\eta, \operatorname{supp}(g)}\right\}_{g \in G}\right) \subset D_{\zeta} .
$$

It is not difficult to prove that $P_{\eta}$ is closed under multiplication. In fact, this easily follows from the fact that $\operatorname{supp}(g) \cup \operatorname{supp}(h)=\operatorname{supp}(g+h)$ for all $g, h \in G$. For more details see [Ci, Lemma 3]. Thus, $P_{\eta}$ is a commutative domain for each $\eta<2^{2^{\omega}}$.

Proposition 3.1. $P_{\eta}$ is short for each $\eta<2^{2^{\omega}}$.

Proof. $\mathbf{R}$ and $\mathbf{N}$ are short so, by Proposition 1.3, $G=S(\mathbf{R}, \mathbf{N})$ is also short. Hence again by Proposition 1.3, it is enough to prove that $E_{\eta, \operatorname{supp}(g)}$ is short for every $g \in G$. But let $S=\bigcup\{\operatorname{supp}(i(r)): r \in \operatorname{supp}(g)\} \subset S\left(2^{\omega}+1, \mathbf{N}\right)$. Let $H$ be a subgroup of $S\left(2^{\omega}+1, \mathbf{N}\right)$ generated by $S$. Thus, $H$ is at most countable, 
hence is short. This implies that $S\left(H, \mathbf{Q}\left(B_{\zeta}\right)\right)$ is short. But $S\left(H, \mathbf{Q}\left(B_{\zeta}\right)\right)$ is evidently isomorphic to the subdomain

$$
D^{\prime}=\left\{h \in S\left(S\left(2^{\omega}+1, \mathbf{N}\right), \mathbf{Q}\left(B_{\zeta}\right)\right): \operatorname{supp}(h) \subset H\right\} \subset E_{\zeta}
$$

and $E_{\eta, \operatorname{supp}(g)} \subset D^{\prime}$. Hence $E_{\eta, \operatorname{supp}(g)}$ is short for every $g \in G$ and hence so is $P_{\eta}$.

Proposition 3.2. The quotient field of $P_{\eta}$ is equal to $F_{\zeta}$ for every $\eta<2^{2^{\omega}}$.

Proof. Let us fix $\eta<2^{2^{\omega}}$, and let $F$ be the quotient field of $P_{\eta}$ in $F_{\zeta}$. It is enough to prove that $D_{\zeta}=S\left(G, K_{\zeta}\right) \subset F$.

First notice that for every $y \in E_{\zeta}$ there exists $r \in \mathbf{R}$ such that $i(r)=y$. This implies that $y \cdot e_{r} \in P_{\eta}$ and so $y=y \cdot e_{r} / e_{r} \in F$. Thus we have proved that $E_{\zeta} \subset F$. So $K_{\zeta}$ as the quotient field of $E_{\zeta}$ is also included in $F$.

This implies that for every $x \in K_{\zeta}$ and $g \in G$ an element $x \cdot e_{g} \in F$. But obviously $D_{\zeta}=S\left(G, K_{\zeta}\right)$ is generated by $\left\{x \cdot e_{g}: x \in K_{\zeta}\right.$ and $\left.g \in G\right\}$, i.e., $D_{\zeta} \subset F$. This finishes the proof of Proposition 3.2.

As a last step to prove our main theorem we have to prove Proposition 3.3. $P_{\xi}$ and $P_{\eta}$ are nonisomorphic for every $\xi<\eta<2^{2^{\omega}}$.

Proof. By Lemma 1.2 we have for every $\eta<2^{2^{\omega}}$,

$$
\begin{aligned}
Q\left(C_{\eta}\right)^{+} & =\operatorname{rad}\left(\mathbf{Q}\left(C_{\eta}\right)\right) \subset \operatorname{rad}\left(P_{\eta}\right) \subset \operatorname{rad}\left(F_{\eta}\right) \cap P_{\eta} \\
& =\mathbf{Q}\left(B_{\zeta}\right)^{+} \cap P_{\eta}=\mathbf{Q}\left(B_{\zeta}\right)^{+} \cap \mathbf{Q}\left(C_{\eta}\right)=\mathbf{Q}\left(C_{\eta}\right)^{+} .
\end{aligned}
$$

In other words

$$
\mathbf{Q}\left(C_{\eta}\right)^{+}=\operatorname{rad}\left(P_{\eta}\right) \text { for every } \eta<2^{2^{\omega}} \text {. }
$$

By way of contradiction let us assume that there exists a field isomorphism $j: P_{\xi} \rightarrow P_{\eta}$ for some $\xi<\eta<2^{2^{\omega}}$. Thus by the above remark,

$$
j\left(\mathbf{Q}\left(C_{\xi}\right)^{+}\right)=j\left(\operatorname{rad}\left(P_{\xi}\right)\right)=\operatorname{rad}\left(P_{\eta}\right)=\mathbf{Q}\left(C_{\eta}\right)^{+} .
$$

Hence $j\left(\mathbf{Q}\left(C_{\xi}\right)\right)=\mathbf{Q}\left(C_{\eta}\right)$, which is impossible since $\mathbf{Q}\left(C_{\xi}\right)$ and $\mathbf{Q}\left(C_{\eta}\right)$ are real closed subfields of $\mathbf{R}$ and are different as $C_{\xi} \neq C_{\eta}$. Thus $P_{\xi}$ and $P_{\eta}$ are nonisomorphic.

This finishes the proof of our main theorem.

Theorem 1. There exists a family $\mathbf{F}$ of power $2^{2^{\omega}}$ of long ordered fields of size $2^{\omega}$ that are pairwise nonisomorphic and such that every field $F$ from $\mathbf{F}$ has $2^{2^{\omega}}$ nonisomorphic short subdomains whose field of quotients is $F$.

\section{Generalizations FOR Higher CARDinals}

Let $\kappa$ be an infinite cardinal. We say that a linearly ordered set is $\kappa$-short if it does not contain any monotonic sequence of length $\kappa^{+}$, and it is $\kappa$-long if it contains a monotone sequence of length $\alpha$ for every ordinal $\alpha<\left(2^{\kappa}\right)^{+}$. 
The following theorem is a generalization of Theorem 1 for arbitrary $\kappa$ replacing $\omega$.

Theorem 2. Let $\kappa$ be an infinite cardinal. Then there exists a family $\mathbf{F}$ of power $2^{2^{\kappa}}$ of $\kappa$-long ordered fields of size $2^{\kappa}$ that are pairwise nonisomorphic and such that every field $F \in \mathbf{F}$ has $2^{2^{\kappa}}$ nonisomorphic $\kappa$-short subdomains whose field of quotients is $F$.

The proof of the Theorem 2 is based on the same concept as that of Theorem 1. However, it also needs some new ideas and thus we sketch its proof.

First we need the following propositions:

Propositions 4.1. There exists a family $\mathbf{X}$ of $2^{2^{\kappa}}$ nonisomorphic linear orderings of cardinality $2^{\kappa}$ each containing an increasing sequence of length $2^{\kappa}+1$.

Proof. Let $P=\left(2^{\kappa}+1\right) \times[0,1]$ be ordered lexicographically. For every $A \subset 2^{\kappa}$ let $X_{A}=\left\{(\xi, r) \in P:\left(\xi \in A \cup\left\{2^{\kappa}\right\}\right) \Rightarrow(r=1)\right\} \subset P$. It is not difficult to see that $\mathbf{X}=\left\{X_{A}: A \subset 2^{\kappa}\right\}$ satisfies our requirements.

For linearly ordered sets $X$ and $Y$ let $X \oplus Y$ be the linearly ordered set $X \cup Y$ such that the orderings on $X$ and $Y$ are preserved and $x<y$ for every $x \in X$ and $y \in Y$. Let us also notice that the family $\mathbf{X}$ from Proposition 4.1 has the following stronger property:

Proposition 4.2. Let $Y_{1}$ and $Y_{2}$ be linearly ordered sets, and let $X_{1}, X_{2} \in \mathbf{X}$ where $\mathbf{X}$ satisfies the conclusion of Proposition 4.1. If $j: X_{1} \oplus Y_{1} \rightarrow X_{2} \oplus Y_{2}$ is an order isomorphism then $X_{1}=X_{2}$ and $j\left(Y_{1}\right)=Y_{2}$.

We also need the following theorem of Todorcevic [To], which is an answer to a problem asked in an earlier version of this paper.

Theorem 3. For every infinite cardinal $\kappa$ there exists a $\kappa$-short linearly ordered set $Y$ of size $2^{\kappa}$ with $2^{2^{\kappa}}$ nonisomorphic rigid linear suborderings of power $2^{\kappa}$.

For the sake of completeness and the fact that [To] may not be widely available, we reproduce the proof of Theorem 3 in $\S 5$ of this paper.

Proof of Theorem 2. Let us fix an infinite cardinal, let $Y$ be a $\kappa$-short linearly ordered set of cardinality $2^{\kappa}$ satisfying Theorem 3 , and let $\mathbf{X}$ be from Proposition 4.1.

For $X \in \mathbf{X}$ let us define $G(X)=S(X \oplus Y, \mathbf{Q})$ and let

$$
K_{X}=\left\{f \in \mathbf{R}^{G(X)}: \operatorname{supp}(f) \text { is decreasingly well ordered in type }<\omega_{1}\right\} .
$$

Lemma 4.3. $K_{X}$ is a real closed $\kappa$-long ordered field of cardinality $2^{\kappa}$ for every $X \in \mathbf{X}$.

Proof. Let us fix $X \in \mathbf{X}$. By Proposition 1.1(3) $G(X)$ is a divisible group. Thus $K_{X}$ is a real closed field (see [A1, Corollary 3.2]). By the choice of $X$ it also contains increasing sequence of length $2^{K}+1$, and the same argument as for Proposition 1.2 shows that $K_{X}$ is $\kappa$-long. The cardinality of $K_{X}$ is obvious. 
Lemma 4.4. The fields $K_{X_{1}}$ and $K_{X_{2}}$ are nonisomorphic for different $X_{1}, X_{2} \in$ $\mathbf{X}$.

Proof. Let us assume that there exists a field isomorphism $j: K_{X_{1}} \rightarrow K_{X_{2}}$ for some $X_{1}, X_{2} \in \mathbf{X}$. We prove that this implies $X_{1}=X_{2}$.

We start with the following:

Claim. There exists an ordered group isomorphism $i: S\left(X_{1} \oplus Y, \mathbf{Q}\right) \rightarrow$ $S\left(X_{2} \oplus Y, \mathbf{Q}\right)$.

Proof of Claim. By definition $G\left(X_{k}\right)=S\left(X_{k} \oplus Y, \mathbf{Q}\right)$. Thus we have to find an ordered group isomorphism $i: G\left(X_{1}\right) \rightarrow G\left(X_{2}\right)$.

Let $\sim$ be the equivalence relation defined by $a \sim b$ if and only if there exist natural numbers $n$ and $m$ such that $a<n \cdot b$ and $b<m \cdot a$, with the equivalence class of $a$ denoted by $[a]$. Then, $K_{X_{k}} / \sim=\left\{[a]: a \in\left(K_{X_{k}}\right)^{+}, a>\right.$ $0\}$ is an ordered group.

Next, notice that $j$ preserves the orderings of $K_{X_{1}}$ and $K_{X_{2}}$ as these fields are real closed. Moreover, $G\left(X_{k}\right)$ is isomorphic, as an ordered group, with the group $K_{X_{k}} / \sim$. But an isomorphism between $K_{X_{1}}$ and $K_{X_{2}}$ induces an isomorphism between $K_{X_{1}} / \sim$ and $K_{X_{2}} / \sim$. This finishes the proof of the claim.

Since $i: S\left(X_{1} \oplus Y, \mathbf{Q}\right) \rightarrow S\left(X_{2} \oplus Y, \mathbf{Q}\right)$ is an ordered group isomorphism, we can repeat the proof the claim to show the existence of an order isomorphism between $S\left(X_{k} \oplus Y, \mathbf{Q}\right) / \sim$ and $X_{k} \oplus Y$ for $k=1,2$ and between $S\left(X_{1} \oplus Y, \mathbf{Q}\right) / \sim$ and $S\left(X_{2} \oplus Y, \mathbf{Q}\right) / \sim$ where $\sim$ is defined similarly as in the claim. This gives us an order isomorphism between $X_{1} \oplus Y$ and $X_{2} \oplus Y$. But by Proposition 4.2, this implies that $X_{1}=X_{2}$. This finishes the proof of Lemma 4.4.

Now for $X \in \mathbf{X}$ let $F_{X}$ be a quotient field of $S\left(S(Y, \mathbf{N}), K_{X}\right)$ and let us define

$$
\mathbf{F}=\left\{F_{X}: X \in \mathbf{X}\right\}
$$

Evidently $F_{X} \supset K_{X}$ is $\kappa$-long. Also, if $F_{X_{1}}$ and $F_{X_{2}}$ are isomorphic for some $X_{1}, X_{2} \in \mathbf{X}$ then, by Lemmas 1.5 and $4.3, K_{X_{1}}$ and $K_{X_{2}}$ are isomorphic as well. But by Lemma 4.4, this implies that $X_{1}=X_{2}$. In other words, different fields from $\mathbf{F}$ are nonisomorphic.

Now let us fix $X \in \mathbf{X}$ and put $F=F_{X}$. To finish the proof of Theorem 2 we have to find $2^{2^{\kappa}}$ nonisomorphic $\kappa$-short subdomains of $F$ for which a field of quotient is $F$.

Let $\mathbf{Z}$ be a family of $2^{2^{\kappa}}$ nonisomorphic subsets of $Y$ of cardinality $2^{\kappa}$. For $Z \in \mathbf{Z}$ let

$$
L_{Z}=\left\{f \in \mathbf{R}^{S(Z, \mathbf{Q})}: \operatorname{supp}(f) \text { is decreasingly well ordered in type }<\omega_{1}\right\} .
$$

As in Lemma 4.3 we conclude that $L_{Z}$ is a real closed field of cardinality $2^{\kappa}$. Notice also that if $T=\left\{f \in L_{Z}: \operatorname{supp}(f)\right.$ has at most one element $\}$ then $L_{Z}$ 
can be identified with decreasing sequences from $T^{<\omega_{1}}$ where $T^{<\omega_{1}}$ is ordered lexicographically. Thus, similarly as in Lemma 4.5, we can show that $L_{Z}$ is $\kappa$-short. Moreover, up to isomorphism, $L_{Z} \subset K_{X}$ as $S(Z, \mathbf{Q})$ is isomorphic to a subgroup of $S(X \oplus Y, \mathbf{Q})$.

Let $i$ be a bijection between $Y$ and $F$, and for a finite set $A \subset Y$ let $E_{A}$ be the subdomain of $F$ generated by $L_{Z} \subset F$ and the set $\{i(r): r \in A\} \subset F$. In particular $E_{\varnothing}=L_{Z}$. Let us define

$$
S_{Z}=S\left(S(Y, \mathbf{N}),\left\{E_{\operatorname{supp}(g)}\right\}_{g \in S(Y, \mathbf{N})}\right) \subset F .
$$

As Proposition 1.3 is true also for $\kappa$-short linear orderings we easily conclude that $S(Y, \mathbf{N})$ is $\kappa$-short. Also, as $E_{\text {supp }(g)}$ is generated by $\kappa$-short $D_{Z}$ and finite numbers of elements, it is not difficult to prove that $E_{\text {supp }(g)}$ is $\kappa$-short for every $g \in S(Y, N)$. Hence $S_{Z}$ is $\kappa$-short.

The proofs that $S_{Z}$ is a subdomain of $F$ and that the quotient field of $S_{Z}$ is $F$ are similar to that presented in $\S 3$ for $\kappa=\omega$ and are left to the reader.

Finally, let $S_{Z_{1}}$ and $S_{Z_{2}}$ be isomorphic for some $Z_{1}, Z_{2} \in \mathbf{Z}$. Then, as in Proposition 3.3, we can prove that $L_{Z_{1}}$ and $L_{Z_{2}}$ are isomorphic. But now, repeating the argument from Lemma 4.4 , we conclude that $Z_{1}$ and $Z_{2}$ are isomorphic, so $Z_{1}=Z_{2}$.

This finishes the proof of the Theorem 2 .

\section{Proof of the Theorem 3}

The purpose of this section is to reproduce the general construction of [To] from which Theorem 3 follows. Familarity with [Je, §1.7] or [Ku, §2.6] is assumed.

Fix a regular uncountable cardinal $\theta$ that is not inaccessible, and let $\lambda<\theta$ be the minimal cardinal with the property $2^{\lambda} \geq \theta$. Let $\lim (\omega)$ be the class of all ordinals of cofinality $\omega$. For each $\delta$ in $\lim (\omega) \cap \theta$ fix a strictly increasing function $x_{\delta}: \omega \rightarrow \delta$ cofinal in $\delta$. Let $\ll$ be a linear ordering of $\theta$ such that $(\theta, \ll)$ is isomorphic to a subset of $\left(\{0,1\}^{\lambda}, \leq_{\operatorname{lex}}\right)$ where $\leq_{\text {lex }}$ stands for the lexicographical order. As in Proposition 1.3 we argue that $(\theta, \ll)$ is $\lambda$-short.

Now for each $S \subset \lim (\omega) \cap \theta$ we define

$$
L(S)=\left\{x_{\delta}: \delta \in S\right\}
$$

ordered lexicographically with respect to $\ll$, i.e.,

$$
x_{\delta}<x_{\gamma} \text { if and only if } x_{\delta}(n) \ll x_{\gamma}(n) \text {, where } n=\min \left\{m: x_{\delta}(m) \neq x_{\gamma}(m)\right\} \text {. }
$$

It is easy to see that

Proposition 5.1. $L(S)$ is $\lambda$-short for every $S \subset \lim (\omega) \cap \theta$. 
We also need the following propositions:

Proposition 5.2. If $S \subset \lim (\omega) \cap \theta$ is stationary then there is $r(S) \subset S$ with $S \backslash r(S)$ being nonstationary such that every nontrivial interval of $L(r(S))$ contains $x_{\delta}$ for a stationary set of $\delta$ 's in $r(S)$.

Proof. Let $S_{0}$ be the set of those $\delta \in S$ such that for some natural number $n$ the set $\left\{\gamma \in S: x_{\delta \mid n} \subset x_{\gamma}\right\}$ is nonstationary. First let us show that $S_{0}$ is nonstationary.

So by way of contradiction, let us assume that $S_{0}$ is stationary. Thus, there exists a stationary set $T \subset S$ such that the number $n$ from the above definition is the same for every $\delta \in T$. Now using the Pressing-Down Lemma $n$-times (see [Ku, Lemma 6.15, p. 80]) for the regressive functions $f_{i}\left(x_{\delta}\right)=x_{\delta}(i)$ we can find a stationary set $T^{\prime} \subset T$ such that $x_{\delta \mid n}=x_{\gamma \mid n}$ for every $\delta, \gamma \in T^{\prime}$. But this implies that for $\delta \in T^{\prime} \subset S_{0}$ the set $\left\{\gamma \in S: x_{\delta \mid n} \subset x_{\gamma}\right\}$ contains $T^{\prime}$, i.e., is stationary. This gives the desired contradiction.

Now for $\delta, \gamma \in S \backslash S_{0}$ let $I(\delta, \gamma)$ denote the set of those $\alpha \in S \backslash S_{0}$ such that $x_{\alpha}$ belongs to the open interval in $L\left(S \backslash S_{0}\right)$ with endpoints $x_{\delta}$ and $x_{\gamma}$. Notice that if $I(\delta, \gamma)$ is nonstationary for some $\delta, \gamma \in S \backslash S_{0}$ then $I(\delta, \gamma)$ is empty. Otherwise, if $x_{\alpha} \in I(\delta, \gamma)$ then for some natural number $n$ the set $\left\{\gamma \in S \backslash S_{0}: x_{\alpha \mid n} \subset x_{\gamma}\right\}$ is a subset of the interval with endpoints $x_{\delta}$ and $x_{\gamma}$, i.e., $x_{\alpha} \in S_{0}$.

Let $S_{1}$ be the set of those $\gamma \in S \backslash S_{0}$ such that for some $\delta<\gamma, \delta \in S \backslash S_{0}$, the set $\left\{\alpha: x_{\alpha} \in I(\delta, \gamma)\right\}$ is nonstationary. If $S_{1}$ is nonstationary then $r(S)=$ $S \backslash\left(S_{0} \cup S_{1}\right)$ satisfies the required properties. So by way of contradiction, let us assume that $S_{1}$ is stationary, and let $f$ be the regressive function $f$ on $S_{1}$ defined by $f(\gamma)=\delta$ where $\delta<\gamma$ is such that $I(\delta, \gamma)$ is empty. By the Pressing-Down Lemma there exists a stationary set $T \subset S_{1}$ such that $f$ is constant on $T$. But this is impossible because at most two of the intervals $I(f(\gamma), \gamma)$ for $\gamma \in T$ would be empty. This finishes the proof of Proposition 5.2.

Proposition 5.3. If $S \subset \lim (\omega) \cap \theta$ is stationary then $L(S)$ is not isomorphic to a subset of $\left(\{0,1\}^{\lambda}, \leq_{\mathrm{lex}}\right)$.

Proof. By the minimality of $\lambda<\theta$ and the fact that $\theta$ is regular, $2^{<\lambda}<\lambda$. Thus, $\left(\{0,1\}^{\lambda}, \leq_{\text {lex }}\right)$ does not contain $\lambda$ disjoint intervals. On the other hand the set $\left\{x_{\delta \mid n}: \delta \in S\right.$ and $\left.n \in \mathbf{N}\right\}$ has cardinality $\theta \geq \lambda$ and, by Proposition 5.2 , for a stationary set of $\delta$ 's the set $\left\{x_{\gamma}: x_{\delta \mid n} \subset x_{\gamma}\right\}$ contains a nonempty interval. Thus, $L(S)$ contains $\lambda$ disjoint nonempty intervals.

Proposition 5.4. If there is an order embedding of $L\left(S_{0}\right)$ into $L\left(S_{1}\right)$ then $S_{0} \backslash S_{1}$ is nonstationary in $\theta$.

Proof. Assume that $S=S_{0} \backslash S_{1}$ is stationary and fix strictly increasing $f: L\left(S_{0}\right)$ $\rightarrow L\left(S_{1}\right)$. Since $f$ is one-to-one, the Pressing-Down Lemma implies that the 
set of those $\delta$ in $S$ for which $f\left(x_{\delta}\right)=x_{\gamma}$, and $\delta \geq \gamma$ (so $\delta>\gamma$, since $\delta \neq \gamma$ ) is nonstationary.

So we may find a stationary set $S^{\prime} \subset S$ such that for every $\delta$ in $S^{\prime}$ if $f\left(x_{\delta}\right)=x_{\gamma}$ then $\delta<\gamma$. Moreover, we can also assume, choosing a subset of $S^{\prime}$, if necessary, that for some natural number $n$ if $f\left(x_{\delta}\right)=x_{\gamma}$ then $x_{\gamma}(n-1)<$ $\delta \leq x_{\gamma}(n)$. Furthermore, applying the Pressing-Down Lemma $n$-times, we can find a stationary set $S^{\prime \prime} \subset S^{\prime}$ and a function $t: n \rightarrow \theta$ such that $t \subset x_{\gamma}$ for every $\gamma$ such that $\delta \in S^{\prime \prime}$ and $f\left(x_{\delta}\right)=x_{\gamma}$. Moreover, we may assume that if $\delta<\delta^{\prime}$ are in $S^{\prime \prime}$ and if $f\left(x_{\delta}\right)=x_{\gamma}$ then $\delta \leq x_{\gamma}(n)<\gamma<\delta^{\prime}$. But this means that the lexicographical ordering of $f\left(L\left(S^{\prime \prime}\right)\right)$ is determined by the $n$th coordinate and therefore, is isomorphic with the subset of $\left(\{0,1\}^{\lambda}, \leq_{\operatorname{lex}}\right)$, which contradicts Proposition 5.3.

As an immediate corollary from Propositions 5.3 and 5.4 we obtain the following conclusion.

Proposition 5.5. Let $S \subset \lim (\omega) \cap \theta$ be stationary in $\theta$. If $r(S) \subset S$ is as in Proposition 5.2 then $L(r(S))$ is rigid.

Now notice that there exist a family $\mathbf{S}_{0}$ of size $2^{\theta}$ of stationary subsets of $\lim (\omega) \cap \theta$ such that symmetric difference $S_{0} \Delta S_{1}$ is stationary for all distinct $S_{0}, S_{1} \in \mathbf{S}_{0}$. This follows from the fact that $\lim (\omega) \cap \theta$ is the union of $\theta$ disjoint stationary sets (see [Je, Lemma 7.6, p. 59]). Put $Y_{\theta}=L(\lim (\omega) \cap \theta)$ and let $\mathbf{S}_{\theta}=\left\{r(S): S \in \mathbf{S}_{0}\right\}$.

If $2^{\kappa}$ is regular then put $\theta=2^{\kappa}$ and $Y=Y_{\theta}$ satisfies the requirements of Theorem 3 as $\left\{L(S): S \in \mathbf{S}_{\theta}\right\}$ is a family of rigid pairwise nonisomorphic subsets of $Y$.

Suppose $2^{\kappa}$ is singular with cofinality $\theta$, and fix a sequence $\theta_{\xi}(\xi<\theta)$ of successor cardinals increasingly converging to $2^{\kappa}$ such that $\theta_{0}>\theta$. For each $\xi$ fix a family $\mathbf{S}_{\theta_{\xi}}$ as above and let $K=\left\{k_{\xi}: \xi<\theta\right\}$ be a suborder of $\left(\{0,1\}^{\kappa}, \leq_{\text {lex }}\right)$. Let $Y$ be an ordering obtained by inserting $L\left(\lim (\omega) \cap \theta_{\xi}\right)$ in place of $k_{\xi}(\xi<\theta)$ in $K$. It is not difficult to see that $Y$ is $\kappa$-short and has cardinality $2^{\kappa}$. Moreover, if for each $\xi<\theta$ we choose $S_{\xi} \in \mathbf{S}_{\theta_{\xi}}$, and taking only $L\left(S_{\xi}\right)$ in place of $k_{\xi}$, we get (compare Proposition 5.5) a rigid linear subordering of $Y$. Since there are $2^{2^{k}}$ different choices of $S_{\xi}$ 's, and different choices give nonisomorphic orders (compare Proposition 5.4), Theorem 3 is proved.

\section{REFERENCES}

[A1] N. Alling, On the existence of real-closed fields that are $\eta_{\alpha}$-sets, Trans. Amer. Math. Soc. 103 (1962), 341-352.

[Ci] K. Ciesielski, Short ordered commutative domain whose quotient field is not short, Algebra Universalis 23 (1987), 1-6.

[Ha] F. Hausdorff, Grundzüge der Mengenlehre, Verlag von Veit, Leipzig, 1914. (German) 
[Je] T. Jech, Set theory, Academic Press, New York, 1978.

[Ku] K. Kunen, Set theory, North-Holland, Amsterdam, 1980.

[La] S. Lang, Algebra, Addison-Wesley, Reading, MA, 1984.

[To] S. Todorcevic, A class of linearly ordered sets, handwritten notes, January 1989.

Department of Mathematics, West Virginia University, Morgantown, West Virginia 26506 